\title{
Glucose and Lactate Levels at Admission as Predictors of In-hospital Mortality
}

\author{
David Sotello $^{1}$, Shengping Yang ${ }^{2}$, Kenneth Nugent ${ }^{3}$ \\ 1. Internal Medicine/Pulmonary and Critical Care Medicine, Texas Tech Health Sciences Center, Lubbock, USA 2. \\ Biostatistics, Pennington Biomedical Research Center, Baton Rouge, USA 3. Internal Medicine/Pulmonary and Critical \\ Care Medicine, Texas Tech University Health Sciences Center, Lubbock, USA
}

Corresponding author: David Sotello, david.sotelloaviles@coxhealth.com

\section{Abstract \\ Objective}

Glucose and lactate levels in patients at the time of admission have been studied in diverse patient groups. Some studies suggest that elevated glucose levels at admission predict worse outcomes. Elevated Lactate levels have also been reported to be directly associated with increased mortality. We wanted to determine if the combination of admission glucose and lactate levels improves the predictability of inpatient mortality and length of stay (LOS).

\section{Methods}

This is a retrospective study. We included all adult patients admitted at an academic medical center from October 1, 2015 to September 30, 2016. We collected basic clinical information, including age, gender, admission glucose and lactate levels, LOS, and mortality. We separated outcomes based on glucose and lactate levels by dividing them into quartiles. We also stratified patients based on normal lactate $(<2.0$ $\mathrm{mmol} / \mathrm{L}$ ), high lactate $(2.0-4.0 \mathrm{mmol} / \mathrm{L})$, and very high lactate $(>4 \mathrm{mmol} / \mathrm{L})$ levels; and on normal glucose (60-140 mg/dl), high glucose (140-200 mg/dl), and very high glucose (>200 mg/dl) levels.

\section{Results}

A total of 5,436 adult patients were included in our study. The median age was 58 years, and $57 \%$ of the patients were male. The median LOS was 6 days, and the overall in-hospital mortality rate was $11 \%$. When the patients were separated in quartiles based on admission glucose values, mortality was higher in the 4 th quartile ( $\geqslant 173 \mathrm{mg} / \mathrm{dL}$ ): $14.87 \%$, probability value $(\mathrm{p}):<0.001$. When the patients were separated in quartiles based on lactate levels, the mortality was higher in the 4 th quartile $(\geqslant 2.23 \mathrm{mmol} / \mathrm{L}): 21.95 \%, \mathrm{p}: 0.001$. When the patients were paired according to normal, high, or very high lactate and glucose levels, the groups that had higher mortality were as follows: normal glucose/very high lactate: $32.43 \%$; high glucose/very high lactate: $34.04 \%$; and very high glucose and very high lactate: $39.15 \%$. The groups with very high glucose and very high lactate had increased odds of mortality when compared with the other groups $(\mathrm{p}:<0.001)$.

Received 10/01/2019

Review began 10/02/2019 Review ended 10/18/2019 Published 10/29/2019

\section{(c) Copyright 2019}

Sotello et al. This is an open access article distributed under the terms of the Creative Commons Attribution License CC-BY 3.0., which permits unrestricted use, distribution, and reproduction in any medium, provided the original author and source are credited.

\section{Conclusions}

Admission glucose and lactate levels provide useful information in the estimation of inpatient mortality. The LOS was shortened in the groups with higher glucose, lactate, or both. The combination of glucose and lactate levels predicted mortality better than either value alone.

Categories: Emergency Medicine, Internal Medicine, Other

Keywords: blood glucose, lactate, in-hospital mortality, in-hospital outcomes

\section{Introduction}

The identification of potential clinical indicators that predict patient outcomes is very important in current medical practice [1,2]. There are multiple laboratory tests, clinical scores, and clinical indices that have different ranges of accuracy, and they may not be applicable in all clinical scenarios $[1,2,3]$. It has been established that elevated lactate levels and glucose levels, independently, are directly related to increased patient morbidity, length of stay (LOS), mortality, etc. [4]. These two basic laboratory tests are readily available in most institutions in developed countries. Their combined use has not been extensively studied, and we hypothesized that their combined use may enhance our ability to identify sicker patients who would require more intensive management to improve their outcomes.

\section{Materials And Methods}

This is a single-center, retrospective study. It included all adults ( $\geqslant 18$ years old) admitted to University Medical Center in Lubbock, Texas, from October 1, 2015 to September 30, 2016, whose admission glucose and lactate levels were recorded. Requests for laboratory tests were based on individual patient management 
decisions. Patients who were $<18$ years of age and $>89$ years of age were excluded. General demographic information was collected. This included age, gender, LOS, and mortality. University Medical Center is a 500-bed referral center in West Texas.

The patients were stratified according to their lactate levels into three categories: lactate levels of $<2$ $\mathrm{mmol} / \mathrm{L}$; lactate levels of 2.0-4.0 $\mathrm{mmol} / \mathrm{L}$; and lactate levels $>4.0 \mathrm{mmol} / \mathrm{L}$. The respective LOS and mortality were calculated for each category. The patients were then classified according to their respective quartiles for their glucose and lactate levels, and their respective LOS and mortality were calculated. Patients were also classified according to their glucose/lactate molar ratios into quartiles, with their LOS and mortality for each quartile subsequently calculated.

Finally, the patients were arbitrarily classified according to their paired glucose and lactate levels. We determined the following categories: normal lactate: <2 mmol/L; high lactate: 2.0-4.0 mmol/L; and very high lactate: $>4 \mathrm{mmol} / \mathrm{L}$. These were paired with glucose levels classified as follows: normal glucose: $60-140$ $\mathrm{mg} / \mathrm{dL}$; high glucose: $140-200 \mathrm{mg} / \mathrm{dL}$; and very high glucose: >200 mg/dL. The respective LOS and mortality were calculated for each group.

Descriptive statistics were used to describe the characteristics of the study cohort. Categorical variables were summarized as frequencies, and continuous variables were summarized using medians and quartiles or percentiles, as appropriate. Univariate logistic regression was used to test if there is a significant association between mortality and assigned categories. Odds ratios (ORs) were calculated to evaluate these associations. Comparisons of LOS across categories were performed using the Kruskal-Wallis rank sum test. The statistically significant level was set at 0.05 . Multiple testing adjustment was not performed. Analyses were performed using SAS software, Windows version 9.3 (SAS Institute, Cary, NC).

\section{Results}

A total of 5,436 patients were included in this study. The median age was 58 years (25th-75th percentile: 4469 years); $57 \%$ of the patients were male. The median LOS was 6 days (25th-75th percentile: $3-11$ days). The overall in-hospital mortality was $11.04 \%$. The distribution of glucose, lactate, and glucose/lactate ratios for representative diagnoses are shown in Table 1.

\begin{tabular}{|c|c|c|c|}
\hline Category & Glucose median* & Lactate median* & Glucose/lactate median* \\
\hline Sepsis $(n=799)$ & $132(105.0,202.5)$ & $1.8(1.2,2.8)$ & $4.54(2.75,7.01)$ \\
\hline Pneumonia (n = 118) & $125(103.0,163.5)$ & $1.3(0.9,1.6)$ & $5.60(4.05,7.96)$ \\
\hline Chronic obstructive pulmonary disease exacerbation $(n=159)$ & $123(108.0,149.5)$ & $1.3(1.0,2.1)$ & $5.26(3.48,7.59)$ \\
\hline Acute kidney failure $(n=81)$ & $116(92.0,165.0)$ & $1.4(1.1,1.9)$ & $5.01(3.62,8.15)$ \\
\hline Urinary tract infection $(n=28)$ & $138(106.8,246.5)$ & $1.1(0.9,1.7)$ & $6.74(5.13,10.19)$ \\
\hline Gastrointestinal bleed ( $n=55)$ & $121(98.5,162.5)$ & $1.7(1.2,2.6)$ & $4.69(2.19,8.01)$ \\
\hline Coronary artery disease $(n=191)$ & $118(95.0,149.0)$ & $1.3(1.0,1.7)$ & $5.17(3.62,7.32)$ \\
\hline Acute heart failure $(n=102)$ & $127(108.3,189.5)$ & $1.4(1.1,1.9)$ & $5.39(3.85,7.85)$ \\
\hline Pancreatitis $(n=30)$ & $144(116.5,236.5)$ & $1.4(0.9,2.1)$ & $6.81(4.23,9.03)$ \\
\hline Acute respiratory failure $(n=211)$ & $130(104.0,191.5)$ & $1.5(1.1,2.3)$ & $5.11(3.38,7.21)$ \\
\hline
\end{tabular}

\section{TABLE 1: Distribution of glucose, lactate levels, and glucose/lactate molar ratios of most} representative diagnoses

${ }^{*}$ Numbers in parentheses represent the 25 th and 75 th percentiles

The patients were stratified according to their lactate levels. Those with a lactate level of $<2.0 \mathrm{mmol} / \mathrm{L}$ had a mortality rate of $7.18 \%$; those with a lactate level between $2.0-4.0 \mathrm{mmol} / \mathrm{L}$ had a mortality of $13.16 \%$; and those with a lactate level of $>4 \mathrm{mmol} / \mathrm{L}$ had a mortality of $36.04 \%$. Specifically, when treated as a continuous predictor, a one-unit increase in lactate was associated with a $32 \%$ increase in the odds of mortality (p: $<0.001$ ) (Table 2). 


\section{Cureus}

\begin{tabular}{|l|l|l|l|l|l|}
\hline Lactate (mmol/L) & Lactate level: $<2.0$ & Lactate level: $2.0-4.0$ & Lactate level: $>4.0$ & All & P-valueף \\
\hline Number & 3,763 & 1,193 & 480 & 5,436 & \\
Male/female & $2,105 / 1,658$ & $707 / 486$ & $284 / 196$ & $3,096 / 2,340$ & 0.077 \\
Median age, years & $60(47,70)$ & $55(39,67)$ & $56(42,67)$ & $58(44,69)$ & $<0.001$ \\
Median LOS, days* & $6(4,11)$ & $6(3,11)$ & $5(2,10)$ & $6(3,11)$ & $<0.001$ \\
Mortality* $^{*}$ & $270(7.18)$ & $157(13.16)$ & $173(36.04)$ & $600(11.04)$ & $<0.001$ \\
\hline
\end{tabular}

\section{TABLE 2: Outcomes based on lactate level}

ๆThe distributions of patient age, LOS, and mortality differ among the lactate percentile groups

*Numbers in parentheses are the 25th and 75 th percentiles; **numbers in parentheses are percentage figures

The patients were next divided into quartiles according to the glucose levels. The mortality rate was as follows: 1st quartile (glucose: $<102 \mathrm{mg} / \mathrm{dl}$ ): 9.44\%; 2nd quartile (glucose: 102-125 mg/dl): 9.26\%; 3rd quartile (glucose: $125-173 \mathrm{mg} / \mathrm{dL}$ ); $10.53 \%$; and 4th quartile (glucose: $\geqslant 173 \mathrm{mg} / \mathrm{dl}$ ): $14.87 \%$. Specifically, when comparing the patients in quartiles 1, 2 and 3, the patients in the 4 th quartile had a $68 \%, 71 \%$, and $49 \%$ increase in the odds of mortality, respectively ( $\mathrm{p}:<0.001)$. The LOS was shorter for those in the 4 th quartile (p: <0.001) (Table 3).

The patients were also divided into quartiles according to the lactate levels. The mortality rate was as follows: 1st quartile (lactate: $<1.05 \mathrm{mmol} / \mathrm{L}$ ): $5.56 \%$; 2nd quartile (lactate: $1.05-1.45 \mathrm{mmol} / \mathrm{L}$ ): $6.83 \%$; 3rd quartile (lactate: $1.45-2.23 \mathrm{mmol} / \mathrm{L}$ ): $9.72 \%$; and 4 th quartile (lactate: $>2.23 \mathrm{mmol} / \mathrm{L}$ ): $21.95 \%$. Compared with patients in quartiles 1,2 and 3 , the patients in the 4 th quartile had a $377 \%, 284 \%$, and $161 \%$ increase in the odds of mortality, respectively ( $\mathrm{p}:<0.001)$. The LOS was shorter for those in the 4 th quartile $(\mathrm{p}:<0.001)$ (Table 3).

When the patients were divided into quartiles by their glucose/lactate molar ratios, the mortality was as follows: 1 st quartile (<3.3): 19.72\%; 2nd quartile (3.3-5.1): 9.35\%; 3rd quartile (5.1-7.5): 8.98\%; and 4th quartile $(>7.5): 6.11 \%$. When comparing the patients in the 1 st quartile glucose/lactate molar ratio, the patients in quartiles 2,3 , and 4 had a $58 \%, 60 \%$, and $74 \%$ decrease in the odds of mortality (p: $<0.001$ ) (Table 3). 


\section{Cureus}

Patient details

Glucose, mg/dl

Q1 (<102) Q2 (102-125)

Q3 (125-173)

Q4 (>173)

P-value

Number

Male/female

1,324

1,360

Median age, years ${ }^{*}$

$704 / 620$

$785 / 575$

1,387

$58(43,68)$

$59(45,71)$

$866 / 521$

1,365

Median LOS, days*

$6(3,11)$

$6(3,11)$

Mortality*

125 (9.44)

$126(9.26)$

$60(46,71)$

$741 / 624$

$<0.001$

Lactate, $\mathrm{mmol} / \mathrm{L}$

Lactate

Q1 (<1.05)

Q2 (1.05-1.45)

$57(44,67)$

$<0.001$

Number

Male/female

1,330

1,376

691/639

$784 / 592$

$6(4,11)$

$5(3,10)$

$<0.001$

146 (10.53)

203 (14.87)

$<0.001$

Median age, years*

$60(47,70)$

$60(46,70)$

$59(45,70)$

$6(4,10)$

$6(4,11)$

$6(3,11)$

$94(6.83)$

133 (9.72)

(1)

Median LOS, days*

$74(5.56)$

$(6.83)$

Glucose/lactate in molar ratios

Glucose/lactate molar ratio*

Q1 (<3.3)

Q2 (3.3-5.1)

Q3 (5.1-7.5)

Q4 (>2.23)

P-value

Number

Male/female

1,359

1,359

$815 / 544$

$804 / 555$

1,359

1,362

Median age, years*

$57(40,67)$

$59(43.5,70)$

$748 / 611$

$812 / 550$

$<0.001$

Median LOS, days*

$6(3,12)$

$6(3,10)$

$60(48,71)$

$55(40,66)$

$<0.001$

Mortality ${ }^{\star \star}$

268 (19.72)

127 (9.35)

$6(4,11)$

$5(3,11)$

$<0.001$

$299(21.95) \quad<0.001$

TABLE 3: Outcomes based on glucose and lactate levels in quartiles

Q: quartiles; LOS: length of stay

*Numbers in parentheses are the 25th and 75 th percentiles; **numbers in parentheses are percentage figures

When the patients were classified according to glucose and lactate levels, the lowest mortality was in the group with normal glucose/lactate levels (6.39\%); the highest mortality was in the groups with normal glucose/very high lactate (32.43\%), high glucose/very high lactate (34.04\%), and very high glucose/very high lactate levels (39.15\%). In the normal glucose category, compared with patients in normal and high lactate groups, patients in the very high lactate group had a $603 \%$ and $212 \%$ increase in the odds of mortality (p: $<0.001$ ), respectively. In the high glucose category, compared with patients in the normal and high lactate groups, patients in the very high lactate group had a $345 \%$ and $295 \%$ increase in the odds of mortality (p: 0.001), respectively. In the very high glucose category, compared with patients in normal and high lactate groups, patients in the very high lactate group had a $897 \%$ and $290 \%$ increase in the odds of mortality (p: $<0.001)$, respectively. In addition, patients in the very high glucose/very high lactate group had the shortest LOS (median: 4; 25th-75th percentiles: 2-8) (Table 4). 


\section{Cureus}

Glucose/lactate categories

Glucose/lactate

groups

$N G, N L$

NG, HL

$N G, V H L \quad H G, N L$

HG, HL

HG, VHL VHG, NL

VHG, HL VHG, VHL

$\begin{array}{llllllllll}\text { Number } & 2,457 & 593 & 185 & 722 & 277 & 94 & 561 & 318 & 189 \\ \text { Male/female } & 1,379 / 1078 & 361 / 232 & 103 / 82 & 420 / 302 & 176 / 101 & 62 / 32 & 292 / 269 & 167 / 151 & 115 / 74 \\ & & 55(37, & 57(46, & 63(51, & 55(38, & 58(43, & 57(44, & 53(43, & 54(40, \\ \text { Age, years* } & 59(46,70) & 67) & 69) & 72) & 66) & 70) & 66) & 66) & 64) \\ \text { LOS, days* } & 6(4,11) & 6(3,11) & 5(3,12) & 6(4,11) & 6(3,11) & 6(2,14) & 6(3,11) & 5(3,10) & 4(2,8) \\ \text { Mortality* } & 157(6.4) & 79(13.3) & 60(32.4) & 75(10.4) & 32(11.5) & 32(34) & 34(6.1) & 45(14.1) & 74(39.1)\end{array}$

\section{TABLE 4: Inpatient mortality comparison between glucose and lactate levels}

NG: normal glucose (60-140 mg/dl); HG: high glucose (140-200 mg/dl); VHG: very high glucose (>200 mg/dl)

$\mathrm{NL}$ : normal lactate (<2 mmol/L); HL: high lactate $(2.0-4.0 \mathrm{~mm} / \mathrm{L})$; VHL: very high lactate $(>4.0 \mathrm{mmol} / \mathrm{L})$

LOS: length of stay; *numbers in parentheses are the 25th and 75th percentiles; **numbers in parentheses are percentage figures

The area under the receiver operating characteristic curves (AUROCs) comparing glucose levels, lactate levels, and glucose/lactate ratios shows a higher AUROCs for lactate levels and glucose/lactate levels when compared to glucose levels alone, indicating better performance of lactate levels and glucose/lactate ratios for predictors for mortality (Figure 1).
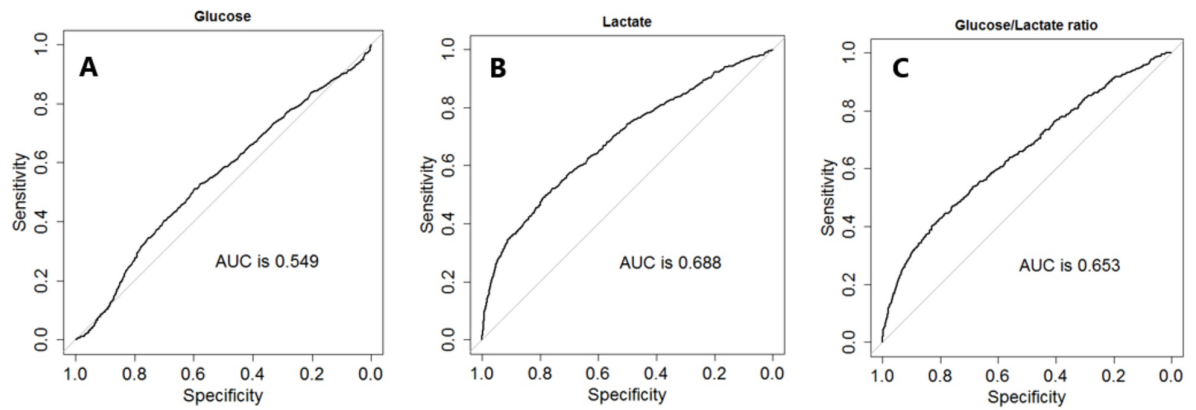

\section{FIGURE 1: Area under the receiver operating characteristic curves (AUROCs) comparing glucose, lactate, and glucose/lactate ratio}

Panel A, B, and C show the AUROCs comparing the performance of glucose levels (panel A), lactate levels (panel B), and glucose/lactate ratios (panel C), showing a better performance of lactate and glucose/lactate ratios when compared with glucose alone as predictor of mortality

\section{Discussion}

In this study, we found that the combination of two well-known markers provides valuable information in the early identification of patients with worse outcomes. The group of patients with a combination of very high glucose and very high lactate had the highest mortality in the study cohort. Our results indicate that this combination provides better identification of the sickest patients when compared to either lactate or glucose levels alone.

It has been well established that elevated lactate levels are associated with increased morbidity, LOS, and mortality $[4,5,6]$. Hyperlactatemia usually occurs when lactate production exceeds lactate clearance. The most common cause is tissue hypoxia, in which lactate is the end product of glycolysis under anaerobic conditions. But this can also occur in disorders unrelated to tissue hypoxia (type B lactic acidosis) [7,8,9]. There is a direct relationship between lactate levels and poor outcomes [4]. Some studies have reported mortality between $75-100 \%$ when lactate levels exceed $>10 \mathrm{mmol} / \mathrm{L}[7,8]$. This association has been studied in several clinical scenarios, especially in septic patients, but may also be applicable in patients in 
other clinical settings, such as emergency room patients, patients with acute coronary syndrome, cardiogenic shock, gastrointestinal bleeding, advanced age, trauma, and postsurgical patients, etc. $[3,4,10,11,12,13]$. Shetty et al. found that the combination of lactate plus quick Sepsis Related Organ Failure Assessment (qSOFA) improved sensitivity in identifying adverse outcomes in suspected septic patients [1]. The optimal lactate cut-off value and the timing of lactate measurement to stratify the sickest patients are still unclear. Larger reductions from baseline lactate levels are associated with better outcomes $[3,7,9,11]$.

The relationship between increased glucose levels and patient outcomes has also been studied, and there is evidence supporting the association of high glucose values with increased morbidity, a longer length of hospitalization, increased readmission, and worse outcomes [14,15,16,17]. Some studies suggest that these relationships exist independently of a known diagnosis of diabetes [15,18-20]. The association of hyperglycemia and worse outcomes seems to be stronger when it occurs in non-diabetic patients [16,20]. Glynn et al. found that among the patients evaluated in the emergency room, abnormal glucose values were associated with increased mortality in non-diabetic patients but not in diabetic patients [2]. Abnormally low glucose levels, especially in the ICU, have also been associated with increased mortality [2]. It is unclear if one or multiple glucose values enhance the ability to triage patients, but Haddadin et al. found that even a single admission of blood glucose could predict increased mortality at 1 year in a mixed cohort of diabetic and non-diabetic patients [19].

These associations may be related to the metabolic changes caused by an acute illness in glucose and lactate metabolism [17,21,22]. It has also been reported that multiple alterations in the glycolysis and gluconeogenesis pathways may contribute to elevated glucose levels [21-23]. Hyperglycemia per se has been associated with immunologic, metabolic, and microvascular changes [18,22]. All of these factors may contribute to a simultaneous elevation of both markers in some critically ill patients.

Very few studies have analyzed combined glucose and lactate levels as predictors of patient outcomes. Kaukonen et al. found that there is an independent association with hyperglycemia and hyperlactatemia and increased mortality. But the association of hyperglycemia disappeared when hyperlactatemia was considered in combination, suggesting that elevated lactate levels may have a stronger association with poor outcomes [21]. Green et al. in their assessment of the association of hyperglycemia and hyperlactatemia found that mortality risk did not increase unless hyperglycemia was associated with hyperlactatemia [23]. Van Vught et al. reported that the association of hyperglycemia and mortality was lost after adjustment for lactate only in non-diabetic patients [18,22]. In our study, we did identify sicker patients when both laboratory test results were used in combination. This may be explained by the different patient populations used in these studies. The previous studies utilized these markers in emergency rooms and ICUs, respectively; we used them in all patients admitted to our hospital.

We found a decreased LOS in the groups with the highest glucose, lactate, or both. The explanation for these results is uncertain. This is in contrast to previously published studies, where elevated lactate levels have been associated with increased LOS [4]. One explanation might be the severity of illness and imminent demise; another explanation might be that these groups included a significant number of patients with diabetic ketoacidosis or hyperosmolar hyperglycemic state who usually have a decreased LOS. The findings between glucose/lactate molar ratios show a novel representation of data that clearly shows that the patients in the first quartile (i.e., those with the highest lactate in proportion to glucose) have increased mortality. Interestingly, the LOS was not affected by this representation of the data.

Some of the strengths of this study include a large population sample, with patients included from multiple clinical settings and services. The authors recognize the limitations of a retrospective study, including the inability to discriminate stress-induced hyperglycemia from diabetes mellitus (lack of a glycosylated hemoglobin value), the lack of serial measurements of lactate and glucose values, and the inability to determine the causes of death.

\section{Conclusions}

The combined use of glucose and lactate levels provides useful information for early-risk stratification to identify those patients with worse clinical outcomes. Patients with very high glucose and very high lactate levels need more evaluation and intensive care.

\section{Additional Information \\ Disclosures}

Human subjects: Consent was obtained by all participants in this study. TTUHSC Lubbock/Odessa Institutional Review Board issued approval L17-024. Approved by our institutional IRB. Animal subjects: All authors have confirmed that this study did not involve animal subjects or tissue. Conflicts of interest: In compliance with the ICMJE uniform disclosure form, all authors declare the following: Payment/services info: All authors have declared that no financial support was received from any organization for the submitted work. Financial relationships: All authors have declared that they have no financial relationships at present or within the previous three years with any organizations that might have an 
interest in the submitted work. Other relationships: All authors have declared that there are no other relationships or activities that could appear to have influenced the submitted work.

\section{References}

1. Shetty A, MacDonald SP, Williams JM, et al.: Lactate $\geqslant 2 \mathrm{mmol} / \mathrm{L}$ plus qSOFA improves utility over qSOFA alone in emergency department patients presenting with suspected sepsis. Emerg Med Australas. 2017, 29:626-634. 10.1111/1742-6723.12894

2. Glynn N, Owens L, Bennett K, Healy ML, Silke B: Glucose as a risk predictor in acute medical emergency admissions. Diabetes Res Clin Pract. 2014, 103:119-126. 10.1016/j.diabres.2013.10.015

3. Cheng HH, Chen FC, Change MW, et al.: Difference between elderly and non-elderly patients in using serum lactate level to predict mortality caused by sepsis in the emergency department. Medicine (Baltimore). 2018, 97:e0209. 10.1097/MD.0000000000010209

4. Chebl RB, El Khuri C, Shami A, Rajha E, Faris N, Bachir R, Abou Dagher G: Serum lactate is an independent predictor of hospital mortality in critically ill patients in the emergency department: a retrospective study. Scand J Trauma Resusc Emerg Med. 2017, 25 :69. Accessed: October 25, 2019: 10.1186/s13049-017-0415-8

5. Soliman HM, Vincent JL: Prognostic value of admission serum lactate concentrations in intensive care unit patients. Acta Clin Belg. 2010, 65:176-181. 10.1179/acb.2010.037

6. Masevicius FD, Rubatto Birri PN, Risso Vazquez A, et al.: Relationship of at admission lactate, unmeasured anions, and chloride to the outcome of critically ill patients . Crit Care Med. 2017, 45:e1233-e1239. 10.1097/CCM.0000000000002730

7. Ferreruela M, Raurich JM, Ayestarán I, Llompart-Pou JA: Hyperlactatemia in ICU patients: incidence, causes and associated mortality. J Crit Care. 2017, 42:200-205. 10.1016/j.jcrc.2017.07.039

8. Haas SA, Lange T, Saugel B, Petzoldt M, Fuhrmann V, Metschke M, Kluge S: Severe hyperlactatemia, lactate clearance and mortality in unselected critically ill patients. Intensive Care Med. 2016, 42:202-210. 10.1007/s00134-015-4127-0

9. Godinjak A, Jusufovic S, Rama A, et al.: Hyperlactatemia and the importance of repeated lactate measurements in critically ill patients. Med Arch. 2017, 71:404-407. 10.5455/medarh.2017.71.404-407

10. Vincent JL, Quintairos E Silva A, Couto L Jr, Taccone FS: The value of blood lactate kinetics in critically ill patients: a systematic review. Crit Care. 2016, 20:257. 10.1186/s13054-016-1403-5

11. Park YJ, Kim DH, Kim SC, et al.: Serum lactate upon emergency department arrival as a predictor of 30-day in-hospital mortality in an unselected population. PLoS One. 2018, 13:e0190519. Accessed: October 25, 2019: 10.1371/journal.pone.0190519

12. Gjesdal G, Braun OÖ, Smith JG, Scherstén F, Tydén P: Blood lactate is a predictor of short-term mortality in patients with myocardial infarction complicated by heart failure but without cardiogenic shock. BMC Cardiovasc Disord. 2018, 18:8. Accessed: October 25, 2019: 10.1186/s12872-018-0744-1

13. Lee SH, Min YW, Bae J, et al.: Lactate parameters predict clinical outcomes in patients with nonvariceal upper gastrointestinal bleeding. J Korean Med Sci. 2017, 32:1820-1827. 10.3346/jkms.2017.32.11.1820

14. Christiansen C, Toft P, Jørgensen HS, Andersen SK, Tønnesen E: Hyperglycaemia and mortality in critically ill patients. A prospective study. Intensive Care Med. 2004, 30:1685-1688. 10.1007/s00134-004-2325-2

15. Evans NR, Dhatariya KK: Assessing the relationship between admission glucose levels, subsequent length of hospital stay, readmission and mortality. Clin Med (Lond). 2012, 12:137-139. 10.7861/clinmedicine.12-2137

16. Martin WG, Galligan J, Simpson S Jr, Greenaway T, Burgess J: Admission blood glucose predicts mortality and length of stay in patients admitted through the emergency department. Intern Med J. 2015, 45:916-924. 10.1111/imj.12841

17. van Beest PA, Spronk PE: Lactate and glucose in critically ill patients: what goes around, comes around . Crit Care Med. 2014, 42:1545-1546. 10.1097/CCM.0000000000000271

18. van Vught LA, Wiewel MA, Klein Klouwenberg PM, et al.: Admission hyperglycemia in critically ill sepsis patients: association with outcome and host response. Crit Care Med. 2016, 44:1338-1346. 10.1097/CCM.0000000000001650

19. Haddadin F, Clark A, Evans N, Dhatariya K: Admission blood glucose helps predict 1 year, but not 2 years, mortality in an unselected cohort of acute general medical admissions. Int J Clin Pract. 2015, 69:643-648. 10.1111/ijcp.12574

20. Kim EJ, Jeong MH, Kim JH, et al.: Clinical impact of admission hyperglycemia on in-hospital mortality in acute myocardial infarction patients. Int J Cardiol. 2017, 236:9-15. 10.1016/j.ijcard.2017.01.095

21. Kaukonen KM, Bailey M, Egi M, Orford N, Glassford NJ, Marik PE, Bellomo R: Stress hyperlactatemia modifies the relationship between stress hyperglycemia and outcome: a retrospective observational study. Crit Care Med. 2014, 42:1379-1385. 10.1097/CCM.0000000000000214

22. Nugent K, Edriss H, Selvan K: Hyperglycemia and outcomes in patients with sepsis. J Thorac Dis. 2016, 8:E575-E577. 10.21037/jtd.2016.05.63

23. Green JP, Berger T, Garg N, et al.: Hyperlactatemia affects the association of hyperglycemia with mortality in nondiabetic adults with sepsis. Acad Emerg Med. 2012, 19:1268-1275. 10.1111/acem.12015 